\title{
Calibración de dos observadores para evaluar restauraciones en una investigación clínica. Aplicación del Índice de Concordancia de KAPPA.
}

Calibration of two observers to evaluate

restorations in a Clinical Research.

Application of the Kappa Index of agreement.

Fecha de Recepción

I de noviembre de 2010
Calibração de dos observadores para

avaliar restaurações em uma Investigação

Clínica. Aplicação do índice de Acordo de Kappa.

Aceptado para su publicación

I4 de marzo de 20 I I
Elena Díaz de Centeno

Profesora Adjunta Asignatura Clínica de Operatoria Dental. FOUNNE.

José Hidalgo

Coordinador Hospital Odontológico Universitario.

FOUNNE.

María Quiroz

Becaria de Investigación SGCyT. FOUNNE.

Rolando Juárez

Profesor Titular Asignatura Fisiología Humana.

FOUNNE.

\section{Resumen}

El propósito del presente trabajo fue establecer el grado de concordancia entre dos observadores, utilizando el Índice de Concordancia de Kappa. Estos observadores evaluarán restauraciones en un estudio prospectivo de cohortes, de tipo clínico, teniendo en cuenta los lineamientos de la odontología basada en evidencias.

La calibración se realizó mediante la observación individual de dos examinadores. Se utilizaron 18 troqueles con restauraciones de resinas compuestas. Los parámetros evaluados en las restauraciones fueron el color, la adaptación marginal, la forma anatómica y la textura superficial. Se calificaron como Alfa, Bravo y Charlie respectivamente, de acuerdo a los criterios clínicos generales Ryge para las restauraciones que se encontraron en estado óptimo, aceptables y para las que requirieron reemplazo. Los resultados se cruzaron y se obtuvieron los pares de frecuencia para cada categoría. El resultado de los análisis dio un grado de acuerdo Bueno $(0,662)$ para el color, Muy Bueno $(0,818)$ para la adaptación Marginal, Bueno $(0,626)$ para la forma anatómica y Bueno $(0,746)$ para la Textura Superficial. La utilización del Índice de Concordancia de Kappa es un buen recurso para obtener confiabilidad, consistencia y validez en los resultados de una investigación clínica.

\section{Palabras claves}

Evaluación Clínica, Validez, Concordancia, Grado de acuerdo. 


\section{Abstract}

The purpose of the present paper, it was to establish the degree of conformity between two observers, using the Index of Kappa's Conformity. The observers will evaluate restorations in a clinical prospective cohort study, taken into account the guidelines of evidence-based practice. The calibration was realized by means of the individual observation. Eighteen dies with restorations of compound resins were used. The parameters evaluated in the restorations of compound resins were the color, the marginal adjustment, the anatomical form and the superficial texture. They were qualify as Alpha, Bravely and Charlie respectively, in agreement to the clinical general criteria Ryge/USPHS, for the restorations that they found in ideal condition, acceptable and for those who needed replacement. The results were compared and the couples of frequency were obtained for every category. Of the analysis it turned out to be a degree agreement.

Key words

Clinical Evaluation, Validity, Conformity, Degree of agreement.

\section{Resumo}

O propósito do trabalho presente era estabelecer o grau de acordo entre dois observadores, enquanto usando o Índice de Acordo de Kappa. Estes observadores avaliarão restaurações em um estudo previdente de coortes, de tipo clínico, se lembrando de os limites da odontologia baseado em evidências. A calibração foi levada a cabo por meio da observação individual de dois examinadores. 18 estampas eram usadas com restaurações de resinas combinação. Os parâmetros avaliados nas restaurações eram a cor, a adaptação marginal, a forma anatômica e a textura superficial. Eles estavam qualificados como Alfa, Valente e Charlie respectivamente, de acordo com as aproximações gerais clínicas Ryge para as restaurações que estavam em bem, estado aceitável e para esses que substituição exigida. Os resultados cruzaram e foram obtidos os pares de frequiência para cada categoria. $O$ resultado das análises deu um grau de acordo Bom (0662) para a cor, Muito Bom (0818) para o Marginal, adaptação Boa (0626) para a forma anatômica e
Boa (0746) para a Textura Superficial. $O$ uso do Índice de Acordo de Kappa é um recurso bom para obter confiança, consistência e validez nos resultados de uma investigação clínica.

\section{Palavras chaves}

Avaliação clínica, Validez, Acordo, Grau de acordó.

\section{Introducción}

Los factores fundamentales que pueden afectar los resultados de una investigación son, la variación del instrumento y la variación del examinador'. El error del examinador, está relacionada con su entrenamiento, su formación, su capacidad ${ }^{2}$.

Hay una fuente importante de error de medidas, en la variabilidad entre los observadores, y como la precisión de las observaciones puede medirse, es necesario destacar la importancia de estimar el grado de concordancia o variabilidad entre ellos. A mayor precisión de las observaciones, mayor validez de los resultados ${ }^{3}$.

El Índice de Concordancia de Kappa, permite medir el grado de acuerdo o concordancia intraevaluador, es decir la evaluación respecto de sí mismo y con otros observadores. Es un coeficiente estadístico que se emplea para cuantificar el grado de acuerdo, corrige el factor azar.

Respecto de su gran aplicabilidad, Campos, Carrascosa y col. ${ }^{4}$ lo utilizaron para medir el acuerdo intra-evaluador, en un estudio para determinar la confiabilidad de la versión en portugués de un cuestionario para diagnosticar desordenes de patologías temporomandibulares.

Por otro lado, Cerda y Villarroel ${ }^{5}$ destacan la utilidad del índice por su simpleza logística, sencillez de análisis estadístico y una amplia aplicabilidad en escenarios clínicos, y dan a conocer los beneficios que tiene en el campo de la medicina pediátrica, mediante un trabajo realizado para calibrar a médicos pediatras en el diagnóstico nutricional de niños, Bouza ${ }^{6}$ coincide y recalca la importancia de estimar el consenso en los estudios biomédicos en los que el operador juega un papel relevante.

El objetivo del presente trabajo fue determinar el grado de acuerdo entre dos observadores mediante el índice de concordancia de Kappa y 
exponer la importancia de su utilidad en el aporte de confiabilidad en los estudios clínicos.

\section{Material y Métodos}

Se utilizaron 18 piezas dentarias humanas, cuyos criterios de inclusión fueron piezas dentarias extraídas recientemente por razones ortodóncicas o periodontales, piezas dentarias con raíces totalmente formadas. Los criterios de exclusión fueron piezas dentarias con caries de más de un tercio de superficie oclusal afectada. Previa limpieza de los restos de tejido orgánico con piedra pómez, brocha y agua se realizaron las preparaciones clase I, II y III de Black, las cuales fueron realizadas por un solo operador. Se utilizaron para la restauración resinas compuestas (ZI00 3M, colores: A2, A3, A3.5, B2, UD).
Fueron polimerizadas con luz halógena (Coltolux 3 de Colteéne) usando la técnica incremental. Los troqueles se realizaron en cubos plásticos con yeso piedra.

Las piezas dentarias restauradas fueron almacenadas después de su extracción, en frascos de vidrio con solución fisiológica a $37^{\circ}$ de temperatura para evitar su deshidratación. Se procedió a enumerar los troqueles en forma consecutiva del I al 18.

La observación se realizó a ojo desnudo, con luz del equipo dental Davi Atlante y explorador tipo Hu Freddy $N^{\circ} 23$.

Las restauraciones fueron valoradas de acuerdo a los Criterios Clínicos Generales Ryge, siendo los parámetros evaluados: color, adaptación marginal, forma anatómica y textura superficial, (Tabla I y II).

Tabla I. Criterios Clínicos de Ryge.

\begin{tabular}{|l|l|}
\hline Alfa & $\begin{array}{l}\text { La restauración presenta excelente condición y se espera que proteja al diente y los } \\
\text { tejidos adyacentes. }\end{array}$ \\
\hline Bravo & $\begin{array}{l}\text { La restauración es aceptable pero muestra uno o mas parámetros defectuosos. Sera } \\
\text { necesario su reemplazo en el futuro. }\end{array}$ \\
\hline Charlie & La restauración es inaceptable y requiere reemplazo. \\
\hline
\end{tabular}

Tabla II. Criterios Clínicos Generales de Ryge por parámetro.

\begin{tabular}{|l|l|l|l|}
\hline Caract. Clínicas & \multicolumn{1}{|c|}{ Alfa } & \multicolumn{1}{c|}{ Bravo } & \multicolumn{1}{c|}{ Charlie } \\
\hline Color & $\begin{array}{l}\text { La restauración coincide } \\
\text { en color y translucidez } \\
\text { con la estructura dentaria } \\
\text { adyacente. }\end{array}$ & $\begin{array}{l}\text { No coincide en color y } \\
\text { translucidez pero esta en } \\
\text { rango aceptable. }\end{array}$ & $\begin{array}{l}\text { La diferencia de color y } \\
\text { translucidez esta fuera del } \\
\text { rango aceptable. }\end{array}$ \\
\hline $\begin{array}{l}\text { Adaptación } \\
\text { Marginal }\end{array}$ & $\begin{array}{l}\text { El explorador no se } \\
\text { traba o se traba en una } \\
\text { sola dirección cuando se } \\
\text { examina a través de la } \\
\text { unión diente-interfase- } \\
\text { restauración. }\end{array}$ & $\begin{array}{l}\text { El explorador cae en una } \\
\text { brecha cuando se examina } \\
\text { en la interfase diente- } \\
\text { restauración. }\end{array}$ & $\begin{array}{l}\text { La dentina o base cavitaria } \\
\text { están expuestas, a lo largo } \\
\text { del margen. }\end{array}$ \\
\hline $\begin{array}{l}\text { Forma } \\
\text { Anatómica }\end{array}$ & $\begin{array}{l}\text { El contorno general de } \\
\text { la restauración sigue el } \\
\text { contorno del diente. }\end{array}$ & $\begin{array}{l}\text { El contorno general de la } \\
\text { restauración no sigue el } \\
\text { contorno del diente. }\end{array}$ & $\begin{array}{l}\text { La restauración presenta } \\
\text { hombro. }\end{array}$ \\
\hline $\begin{array}{l}\text { Textura } \\
\text { Superficial }\end{array}$ & $\begin{array}{l}\text { La superficie de la } \\
\text { restauración no presenta } \\
\text { defectos superficiales }\end{array}$ & $\begin{array}{l}\text { La superficie de la } \\
\text { restauración presenta } \\
\text { mínimos defectos } \\
\text { superficiales. }\end{array}$ & $\begin{array}{l}\text { La superficie de la } \\
\text { restauración presenta } \\
\text { severos defectos } \\
\text { superficiales. }\end{array}$ \\
\hline
\end{tabular}


Los datos registrados fueron volcados en dos hojas de cálculos (Excel), conteniendo en las columnas las variables a observar $y$ en las filas la numeración de los troqueles, donde cada examinador registró su observación en forma independiente.

Se obtuvieron los pares de frecuencia para cada categoría, luego se analizaron mediante la calculadora on- line correspondiente al índice de Kappa $^{(2)}$.

Los parámetros determinados para el grado de acuerdo se consideran 0,2 como Malo y 0,8 como Muy Bueno.

\section{Resultados}

Se obtuvo un grado de acuerdo Bueno $(0,662)$ para el color, cuyo acuerdo fue en II de las 18 unidades evaluadas, 7 con criterio bravo. Muy Bueno (0.818) para la adaptación marginal, con 14 coincidencias en la evaluación, 9 de ellos con criterio bravo. Bueno (0.626) para la forma anatómica con 14 coincidencias entre los evaluadores, 8 con criterio Charlie y Bueno $(0,746)$ con I5 acuerdos entre los observadores, I I con criterio Bravo, para la textura superficial, (Tabla III).

Tabla III. Grado de Acuerdo establecidos por Landis y Koch.

\begin{tabular}{|c|c|}
\hline KAPPA & Grado de Acuerdo \\
\hline$<0$ & Sin acuerdo \\
\hline $0-0,2$ & Insignificante \\
\hline $0,2-0,4$ & Bajo \\
\hline $0,4-0,6$ & Moderado \\
\hline $0,6-0,8$ & Bueno \\
\hline $0,8-1$ & Muy Bueno \\
\hline
\end{tabular}

\section{Discusión}

En la literatura internacional, son pocos los artículos científicos de odontología rehabilitadora que utilizan o señalan algún método confiable para medir o cuantificar la concordancia entre sus clínicos.

Así, en un estudio de monitoreo de restauraciones de amalgama y resinas, Moncada y col..$^{(7)}$ alcanzaron un valor o grado de acuerdo bueno $(0,76)$; asimismo, en la evaluación de restauraciones de resinas de clase I y II en molares temporarios, Pereira Alves dos Santos y col. ${ }^{(8)}$ obtuvieron un valor muy bueno $(0,85)$, concordando con el buen grado alcanzado en nuestro trabajo.

Almerich Silla y Montiel Company ${ }^{(9)}$ obtuvieron un grado muy bueno (0.85) para tres de los 6 odontólogos que se calibraron con el fin de realizar las exploraciones clínicas, permitiendo de esta manera dar más fiabilidad y validez a su estudio. Resultados similares obtuvieron Martignon Biermann y col. ${ }^{(10)}$ en la calibración realizada para llevar adelante su trabajo de evaluación de tres técnicas operatorias, alcanzando 0.8 para los criterios mayores y 0.5 para los criterios menores establecidos por Cvar y Ryge, modificados por Qvist.

\section{Conclusiones}

La facilidad de cálculo como su clara interpretación, hacen del índice de Kappa una herramienta fundamental en la calibración de observadores para una investigación clínica, siendo de gran valor en su aporte para la fiabilidad de los resultados. 


\section{Bibliografía}

I. Pita Fernández S, Pértegas Díaz S. La Fiabilidad de las mediciones clínicas: el análisis de la concordancia para variables numéricas. Unidad de Epidemiología Clínica y Bioestadística. [Revista en línea] 2005. [ actualizado 12 de enero de 2004; acceso 16 de Septiembrede2009]; I-II. Disponible en: http://www.fisterra.com/mbe/ investiga/conc_numerica/conc_numerica2.pdf

2. Molinero LM. Medidas de concordancia para variables cualitativas. Asociación de la sociedad española de hipertensión, Liga española para la lucha contra la hipertensión arterial [sede Web]. España; Septiembre 200I [actualizado Diciembre 200I; acceso 22 de Septiembre de 2009]. Disponible en: http://www.seh-lelha.org/ concor2.htm\#TOP

3. López de Ulibarri Galparsoro I, Pita Fernández S. Medidas de Concordancia: el índice de Kappa. Cad Aten Primaria [Revista en línea] 1999 [acceso 19 de septiembre de 2009]; 6: 169 - 17I. Disponible en: http://www.fisterra.com/mbe/investiga/kappa/ kappa2.pdf

4. Campos JADB, Carrascosa AC, Loffredo LCM, Faria JB. Consistência interna e reprodutibilidade da versão em português do critério de diagnóstico na pesquisa para desordens temporomandibulares (RDC/TMD - ElXO II). Rev Bras Fisioter 2007; II (6): 45I - 459.

5. CerdaJ,Villarroel L. Evaluación de la Concordancia inter-observador en investigación pediátrica: Coeficiente de Kappa. Rev Chil Pediatr 2008; 79 (I): 54 - 58.

6. Bouza $\mathrm{CN}$. Evaluación del Consenso entre expertos: Índices del tipo Kappa, Modelosy Propiedades. Rev. Invest. Operacional. [Revista en línea] 2005 [acceso 19 de septiembre de 2009]; 26 (I): 39-58. Disponible en: http://rev-inv-ope.univ-parisl.fr/ files/26105/IO-26105-5.pdf

7. Moncada G. Fernandez E, Martín J, Caro MG, Caamaño C, Mjor I, Gordan V. Longevidad y Causas de Fracaso de Restauraciones de Amalgama y Resina Compuesta. Revista Dental de Chile 2007; 99 (3): 8 - 16.

8. Pereira Alves dos Santos M, Passos M, Ronir Ragio L, Lucianne Cople M. A Randomized Tiail of Resin - Based Restorations in Class I and Class II Beveled preparations in Primary Molars: 24-Month Results. J Am Dent Assoc. 2009; 140 (2): $156-166$.

9. Almerich Silla J, Montiel Company J. Oral health survey of the child population in the Valencia Region of Spain (2004). Med Oral Patol Oral Cir Bucal 2006; II: 369 - 8I.
10. Martignon Biermann S, Téllez Merchan M, RuizJA, Padilla Castañeda AC, López SJ, Gómez J, Marín LM. Desempeño Clínico de tres técnicas operatorias en preescolares de Bogotá: Estudio Clínico Aleatorizado. Rev Col Invest Odont [revista en Internet]* 20 I0. [acceso II de octubre de 2010]; I (2). Disponible en: http://blog.rcio.org/index. $\mathrm{php/rcio/article/view/I9.}$ 